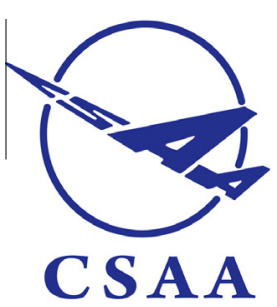

Chinese Society of Aeronautics and Astronautics \& Beihang University

Chinese Journal of Aeronautics

cja@buaa.edu.cn

www.sciencedirect.com

\title{
Acoustic measurements of models of military style supersonic nozzle jets
}

\author{
Ching-Wen Kuo ${ }^{a, *}$, Jérémy Veltin ${ }^{\text {b }}$, Dennis K. McLaughlin ${ }^{a}$ \\ a Department of Aerospace Engineering, The Pennsylvania State University, University Park, PA 16802, USA \\ b TNO Science and Industry, Delft AD 2600, Netherlands
}

Received 29 May 2013; revised 31 July 2013; accepted 5 November 2013

Available online 19 December 2013

\section{KEYWORDS}

Aeroacoustics;

Acoustic noise measurement;

Jet noise;

Jets;

Nozzle;

Supersonic flow

\begin{abstract}
Modern military aircraft jet engines are designed with variable-geometry nozzles to provide optimal thrust in different operating conditions, depending on the flight envelope. However, acoustic measurements for such nozzles are scarce, due to the cost involved in making fullscale measurements and the lack of details about the exact geometries of these nozzles. Thus the present effort at Pennsylvania State University (PSU) in partnership with GE Aviation and the NASA Glenn Research Center is aiming to study and characterize the acoustic field produced by supersonic jets issuing from converging-diverging military style nozzles, and to identify and test promising noise reduction techniques. An equally important objective is to develop methodology for using data obtained from small- and moderate-scale experiments to reliably predict the full-scale engine noise. The experimental results presented show reasonable agreement between small-scale and medium-scale jets, as well as between heated jets and heat-simulated ones.
\end{abstract}

(C) 2014 Production and hosting by Elsevier Ltd. on behalf of CSAA \& BUAA.

\section{Introduction}

Military aircraft have engines with noise characteristics much louder than civilian aircraft, due to their very low bypass ratios and high exit temperatures and velocities of the jets. The resulted increased noise poses a health threat to ground crews as well as causing an annoyance to communities in the vicinity of military airbases. This has led to the development of noise

\footnotetext{
* Corresponding author. Tel.: +1 8143084102.

E-mail addresses: cxk425@psu.edu, cxk425@gmail.com (C.-W. Kuo).

Peer review under responsibility of Editorial Committee of CJA.

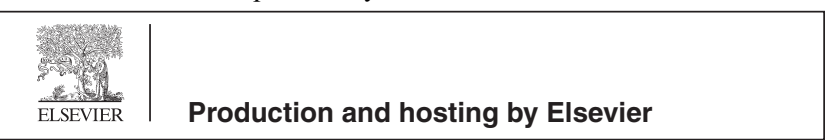

suppression mechanisms that involve new nozzle design concepts such as chevrons, corrugations, beveled nozzles, and other non-axisymmetric geometries. One idea behind noise reduction concepts is to increase the mixing rate between the jet potential core and the surrounding air flow to shorten the length of the high turbulence, noise producing region. A possible way to achieve this is to alter the nozzle geometry at the exit plane in a way that it results in an increase in the turbulence within the shear layer surrounding the potential core. Chevrons and corrugations are examples of such designs. However, noise suppression can also be achieved by departing from the traditional axisymmetric geometries. For example, rectangular and elliptic nozzles are known for producing acoustic spectra with amplitude that depends on the azimuthal angle. Both of these nozzle designs produce decreased acoustic levels in the major axis plane compared to a round nozzle with an equivalent exit area, but an increased overall sound 
pressure level (OASPL) in the minor axis plane. Similarly, beveled nozzles produce an orientation of the acoustic field that results in decreased sound at some azimuthal angles.

Modern military aircraft jet engines are designed to provide optimal thrust in different operating conditions, depending on the flight envelope. To satisfy this requirement, variable throat areas were deployed in the SR-71 "Blackbird" in order to increase the overall performance. A variable-geometry nozzle was also used in the F-14 "Tomcat", improving the overall engine efficiency. Thrust-vectoring rectangular nozzles were also developed and installed in the F-22 "Raptor". All these novel concepts increase the nozzle complexity and the manufacturing cost while having a non-negligible effect on the radiated sound. The database of acoustic measurements for such nozzles is scarce, due to the cost involved in making full-scale measurements and the lack of details about the exact geometries of these nozzles. A clear assessment of the noise generation of jets issuing from these kinds of nozzles needs to be undertaken in order to accurately predict the impact on ground crews and communities and develop efficient noise reduction techniques.

This study is part of an effort led by Pennsylvania State University (PSU) with partners involving GE Aviation and the NASA Glenn Research Center. The aim of the overall project is to study and characterize the acoustic field produced by supersonic jets issuing from converging-diverging military style nozzles, and to identify and test promising noise reduction techniques. An equally important objective was to develop methodology for using data obtained from testing at small and moderate scales, supported by computations, to reliably predict the full-scale engine noises. Therefore, the first step of this project was to conduct and compare small- and medium-scale experiments with nozzles representative of military jet engine exhaust nozzles. Comparisons across scales with data obtained in different facilities would provide confidence in the quality of the measurements performed, and in the ability of the methodology to extrapolate subscale data to full-size aircraft. The second stage is to conduct similar experiments with the addition of chevrons with various detailed designs onto the jet exhaust. Eventually, comparison with flight data obtained with aircraft such as an F-16XL or an F-18 will be performed. This paper focuses solely on the first step of the study: making extensive comparisons between small-scale measurements performed at PSU and medium-scale measurements gathered at the NASA Glenn Research Center, in order to determine whether small-scale and heat-simulated jets can accurately simulate the acoustics issuing from moderate-scale and hot jets.

\section{Technical approach}

\subsection{Description of the experimental facility and instrumentation}

The small-scale experiments presented in this study were conducted in the high speed small-scale jet noise facility at PSU, shown in Fig. 1(a). High-pressure air is drawn from a tank,

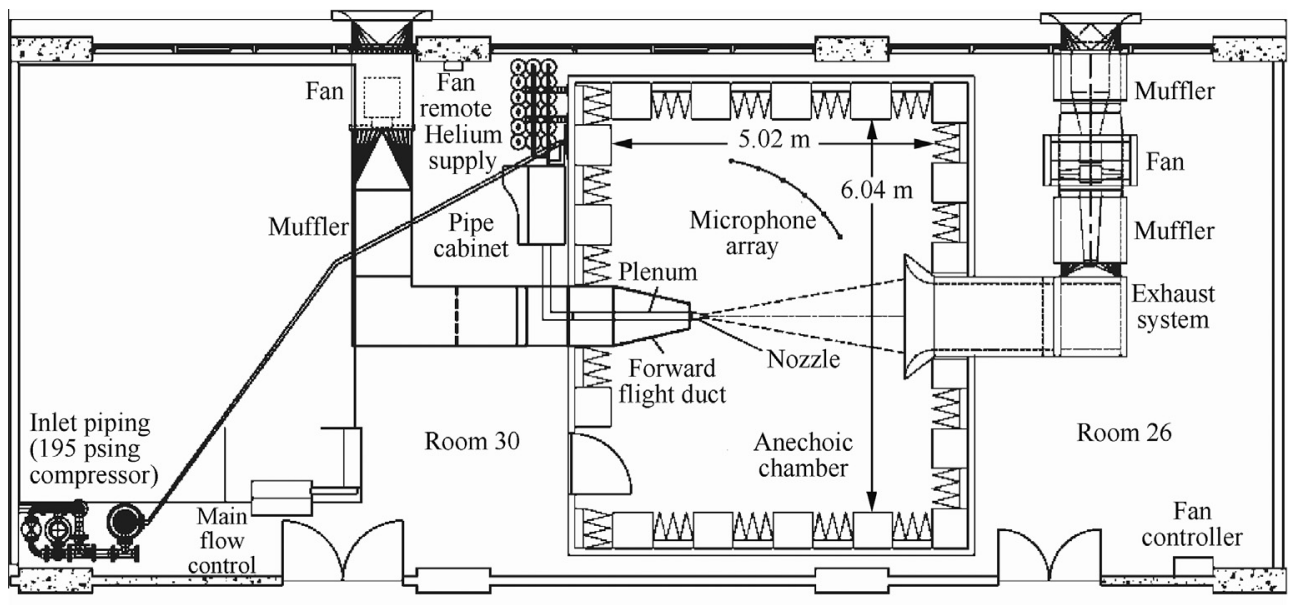

(a) Schematic of the PSU high speed jet noise facility
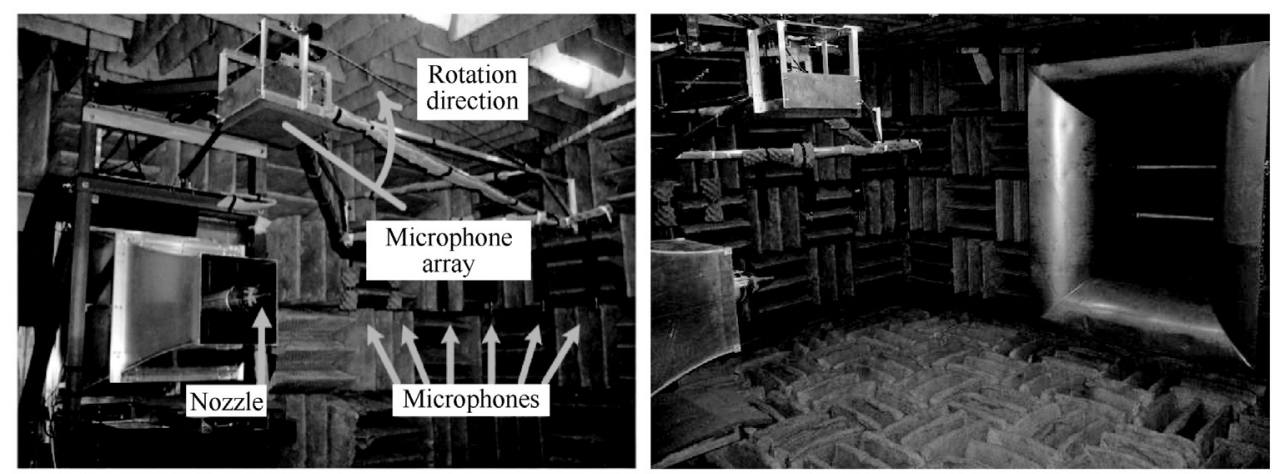

(b) Photograph of the PSU high speed jet noise facility

Fig. 1 Schematic and photographs of the PSU high speed jet noise facility. 
pressurized by a CS-121 compressor combined with a KAD-370 air dryer both manufactured by Kaeser compressors. The air flow is regulated via pressure regulators and control valves located in a piping cabinet before being fed to a plenum and delivered to the jet nozzle issuing into the anechoic chamber. A pitot probe is embedded in the middle section of the plenum to provide, via a pressure transducer, the total pressure upstream of the nozzle. Simulation of heated jets is achieved by mixing helium with the air and discussed in more detail later. The partial pressures of the helium-air mixture are regulated in the piping cabinet. This follows a procedure developed by Doty and McLaughlin ${ }^{1}$ over 10 years ago.

The anechoic chamber is a $5.02 \mathrm{~m} \times 6.04 \mathrm{~m} \times 2.8 \mathrm{~m}$ room covered with fiberglass wedges and with an approximate cut-off frequency of $250 \mathrm{~Hz}$. An exhaust fan installed in the downstream direction of the nozzle collects the jet exhaust and prevents possible helium accumulation in the anechoic chamber.

Acoustic measurements are performed using six microphones, hanging from a boom that extends from the plenum stand, as can be seen in the image of Fig. 1(b). The microphone array can freely rotate around a point located at the center of the nozzle exit plane. The microphones are positioned at a grazing incidence to the jet and equally spaced by $10^{\circ}$. The physical distance, $R$, from each microphone to the nozzle exit is around $1.8 \mathrm{~m}$. This distance is sufficient to ensure that the microphones are in the far field when testing nozzles up to $1.8 \mathrm{~cm}$ in diameter. The microphones used are $3.175 \mathrm{~mm}$ pressure-field microphones, Type 4138 from Brüel and Kjaer (B\&K), and type 40DP from GRAS.

In addition to acoustic measurements of the jets, schlieren visualization of the flow is performed via a conventional Ztype schlieren setup. Detailed descriptions of the setup can be found in the work of Veltin and McLaughlin. ${ }^{2}$

\subsection{Data acquisition}

A flow chart of the data acquisition process is shown in Fig. 2. The microphone calibration is performed with a $\mathrm{B} \& \mathrm{~K}$ acoustic calibrator, model 4231, and the microphone calibration constants are recorded to provide the conversion from the measured voltages to the equivalent pressures. The analog time-domain signals from the microphones are routed through a Nexus, B\&K signal conditioner or a GRAS model 12AN power module and then amplified and filtered for anti-aliasing, thus enabling their accurate digital conversions in the subsequent acquisition. A high-pass filter is also set to
$500 \mathrm{~Hz}$, removing any undesirable low-frequency noise that could contaminate the data. A PCI-6123 National Instruments DAQ board acquires the time-domain data which are then stored in binary files. The sampling rate is set at $300 \mathrm{kHz}$ and 102400-409600 data points are collected. The reduced dataset is used for the helium-air mixture jets in order to reduce the amount of helium used during an experiment. The raw data are split into 1024 or 4096 point segments and a Hanning window is applied with $50 \%$ overlap between each window. The fast Fourier transform (FFT) is calculated in each window and an averaged value is calculated from the 199 segments. This yields the power spectral density (PSD) with a frequency resolution of $74 \mathrm{~Hz}$, which is then converted to a decibel $(\mathrm{dB})$ scale using a reference pressure of $20 \mu \mathrm{Pa}$. Three corrections are then applied to the raw sound pressure level (SPL) to compute the lossless SPL as described by Kuo et al. ${ }^{3,4}$ The frequency resolution of the PSU spectra shown in this paper is plotted between $500 \mathrm{~Hz}$ and $100 \mathrm{kHz}$. Finally, the spectra are non-dimensionalized to SPL per unit Strouhal number St. Eq. (1) summarizes the different steps that lead to the SPL per unit Strouhal number.

$$
\begin{aligned}
\underbrace{\operatorname{SPL}(S t)}_{\text {Lossless spectrum }}= & \underbrace{\operatorname{SPL}_{\text {raw }}(f)}_{\text {Raw spectrum }}-\underbrace{\Delta C_{\text {act }}(f)-\Delta C_{\mathrm{ff}}(f)}_{\text {Microphone corrections }}+\underbrace{\Delta C_{\text {atm }}(f)}_{\text {Atmospheric correction }} \\
& +\underbrace{10 \times \lg f_{\mathrm{c}}}_{\text {Strouhal number scaling }}
\end{aligned}
$$

The $\Delta C$ 's are the corrections (in dB's) of the measured signals attributed to the actuator response $\left(\Delta C_{\text {act }}\right)$ and acoustic free field response $\left(\Delta C_{\mathrm{ff}}\right)$ (of the microphones) and accounting for the atmospheric propagation attenuation $\left(\Delta C_{\mathrm{atm}}\right)$ (due to viscous and relaxation effects). The Strouhal number is defined as $S t=f \mid f_{\mathrm{c}}$, where $f_{\mathrm{c}}$ is the characteristic frequency of the jet defined by $f_{\mathrm{c}}=U_{\mathrm{j}} / D_{\mathrm{j}}$, in which $U_{\mathrm{j}}$ and $D_{\mathrm{j}}$ are the fully-expanded Mach number and the diameter of the jet plume, respectively.

From the SPL, given at intervals of $\Delta f$, the OASPL is calculated from the following formula:

$$
\begin{aligned}
\text { OASPL } & =10 \lg \left[\Delta f \sum 10^{\left.\left(\frac{\operatorname{SPL}(f)}{10}\right)\right]}\right. \\
& =10 \lg \left[\Delta S t \sum 10^{\left(\frac{\operatorname{SPL}(S t)}{10}\right)}\right] .
\end{aligned}
$$

\subsection{Heated jet simulation}

In order to produce acoustic measurements that can be directly compared to aircraft engine measurements, the temperature of

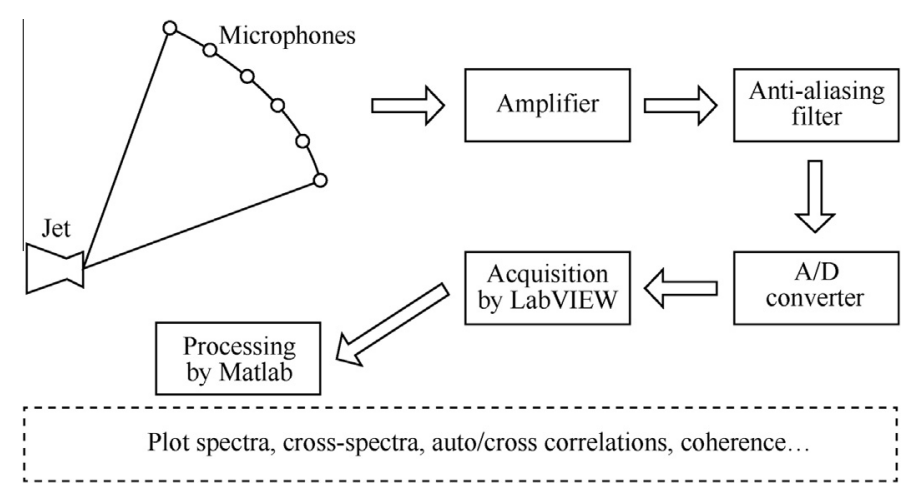

Fig. 2 Flow chart of the data acquisition process. 
the jet is an important parameter that needs to be replicated. A hotter jet results in different acoustical characteristics, due to an increase in jet exit velocity and a decrease in jet density. Actually heating the air that exhausts through the nozzles is done in facilities such as the one used at the NASA Glenn Research Center. ${ }^{5}$ However, it requires an extensive amount of power and infrastructure, raising the overall operating cost of the facility. In order to simulate the flow and acoustic properties of a hot jet, it was shown by Doty and McLaughlin ${ }^{1}$ and by Papamoschou ${ }^{6}$ that a mixture of helium and air could be used. Kinzie and McLaughlin ${ }^{7}$ have demonstrated that the mixture of helium and air is able to replicate the dominant noise characteristics of actual heated jets. The features of heated jets are lowered density and increased acoustic velocity (for a given nozzle pressure ratio). Both of these can be achieved by the addition of helium since it has a significantly lower density than air resulting in a greater gas constant $(R)$, resulting, for a given temperature $(T)$ and Mach number $(M a)$, in a greater acoustic velocity. However, both parameters, the acoustic velocity $a$ and the density $\rho_{\mathrm{j}}$, can not be precisely matched simultaneously. Two different matching methods were introduced by Doty and McLaughlin ${ }^{1}$ and are briefly described here.

The first methodology consists of matching the acoustic velocity between a helium-air mixture and a corresponding hot air jet.

$a_{\text {heated }}=\sqrt{\gamma R T_{\mathrm{j}}} \leftrightarrow a_{\text {mix }}=\sqrt{\gamma_{\text {mix }} R_{\text {mix }} T_{\text {jmix }}}$

where $\gamma_{\text {mix }}$ (the ratio of specific heats) and $R_{\text {mix }}$ are dependent on the helium concentration. The proper molar mass of helium can easily be calculated in order to equate $a_{\text {heated }}$ and $a_{\text {mix }}$. From there, the partial pressure of helium and air can be computed and the pressure regulators were adjusted for the experiment.

The second method consists of matching the density between a heated jet and a helium-air mixture jet, as shown below.

$$
\begin{aligned}
\left(\frac{\rho_{\mathrm{j}}}{\rho_{\infty}}\right)_{\text {heated }} & =\left(\frac{T_{\infty}}{T_{\mathrm{j}}}\right)_{\text {heated }} \leftrightarrow \frac{\rho_{\text {jmix }}}{\rho_{\infty}}=\frac{T_{\infty} R_{\infty}}{T_{\text {jmix }} R_{\text {mix }}} \\
& =\frac{R_{\infty}}{R_{\text {mix }}}\left(1+\frac{\gamma_{\text {mix }}-1}{2} M a^{2}\right)
\end{aligned}
$$

The molar mass of helium required to equate $\rho_{\text {jmix }}$ with $\rho_{\mathrm{j}}$ can be calculated, from which the partial pressure of helium is once again derived. The two matching methods typically lead to slightly different values for the partial pressures of the mixture. However, experimental results ${ }^{1,6}$ showed that the two methods resulted in acoustic spectra in agreement within $1 \mathrm{~dB}$. The PSU experiments were all conducted via using the acoustic velocity matching method.

\subsection{Model geometry of military style supersonic nozzle}

The experimental results presented in this study focus on the acoustic properties of jets issuing from military style nozzles representative of the exhaust of aircraft engines of the F414 family. The exact inner contours are the property of General Electric (GE) and were provided under a contract for the Strategic Environmental Research and Development Program (SERDP). While such military engines possess nozzles with variable geometries adapting to different flight regimes, for this specific research, three nozzles with different exit-to-throat area ratios were used, as specified by GE Aviation. These nozzles are designed with a multi-faceted inside conical contour and the main characteristics of the three nozzles are given in Table 1, including their exit-to-throat area ratios (AR) and exit diameters. The design Mach number $M a_{\mathrm{d}}$ is a convenient identifier used to show the Mach number that would result for perfectly expanded jet flow expanding through the design AR. At PSU, the nozzles were fabricated via a rapid prototyping technique (stereolithography). Fig. 3 shows the schematics of one of the nozzles used at PSU and a nozzle of a similar inner geometry but a larger scale built by GE Aviation and delivered to NASA for the medium-scale experiments conducted at the NASA Glenn Research Center.

\subsection{NASA Glenn facility and measurements overview}

Comparisons are shown in this research with acoustic measurements from similar nozzles at a larger scale acquired in the AeroAcoustic Propulsion Laboratory (AAPL) at the NASA Glenn Research Center. This facility is a hot jet acoustic rig located in a $19.8 \mathrm{~m}$ radius anechoic geodesic hemispherical dome. A microphone arc is mounted at the top of the dome with 24 microphones separated by $5^{\circ}$ increments, and the height of the microphones is $18.3 \mathrm{~m}$ measured from the floor. Jet diameters up to $12.7 \mathrm{~cm}$ are typically used in this facility, with the capability to use a co-flow to simulate jets with a small bypass ratio. Extensive descriptions and qualification measurements of this facility can be found in the work of Bridges and Brown. ${ }^{5}$ As can be seen in Fig. 3, the pressures in both the annular and core sections of the plenum are set to the same value, but the annular flow is kept unheated during hot jet measurements, in order to prevent excessive heating of the nozzle.

\subsection{Data comparison procedure}

The experimental data acquired at PSU are processed into lossless spectra per unit Strouhal number to make comparison easier across scales. The measurements are made at a distance

Table 1 GE nozzle descriptions.

\begin{tabular}{lllll}
\hline AR & $M a_{\mathrm{d}}$ & $\begin{array}{l}\text { PSU GE nozzle equivalent diameter at } \\
\text { exit plane }(\mathrm{cm})\end{array}$ & $\begin{array}{l}\text { NASA GE nozzle equivalent diameter } \\
\text { at exit plane }(\mathrm{cm})\end{array}$ & $\begin{array}{l}\text { Scaling } \\
\text { factor }\end{array}$ \\
\hline 1.067 & 1.3 & 1.63 & 11.69 & $1 / 7.16$ \\
1.180 & 1.5 & 1.72 & 12.29 & $1 / 7.16$ \\
1.295 & 1.65 & 1.80 & 12.87 & $1 / 7.16$ \\
\hline${ }^{*}$ Represents scaling factor compares between PSU and NASA. & &
\end{tabular}




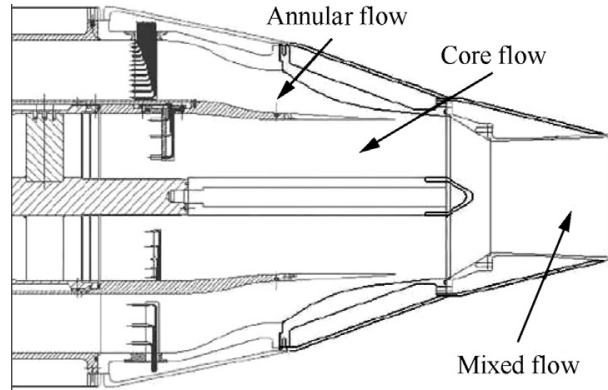

(a) Schematics of one of the nozzles used at PSU

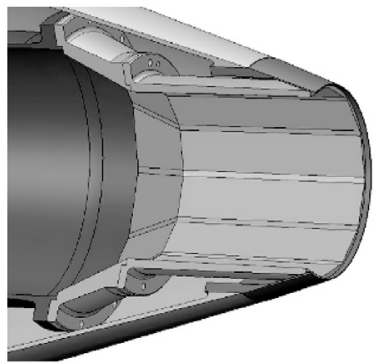

(b) GE nozzle at NASA

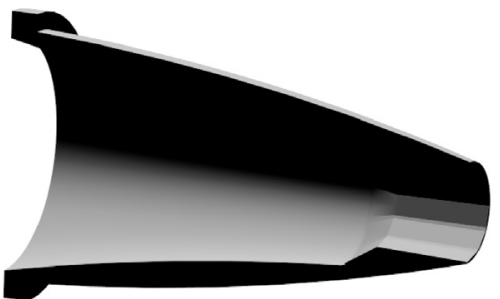

(c) GE nozzle at PSU

Fig. 3 Schematics of GE nozzle with AR $=1.18$, NASA (top), PSU (bottom).

close to $R_{1}=100 \mathrm{D}\left(R_{1}\right.$ : physical distance of the microphones from the nozzle exit, $D$ : diameter of nozzle at exit plane) for the GE nozzles whose exit diameters are about $1.8 \mathrm{~cm}$. The measurements for the smaller contoured converging-diverging (CD) nozzle are made at approximately $R_{1}=144 \mathrm{D}$. Following processing of the resulted data were (back) propagated to $R_{\text {prop }}=100 D$ ( $R_{\text {prop }}$ : propagated distance of resulted data) assuming spherical spreading of the acoustic field. This "back" propagated SPL is determined from:

$\operatorname{SPL}\left(S t, R_{\text {prop }}\right)=\operatorname{SPL}\left(S t, R_{1}\right)+20 \times \lg \left(R_{1} / R_{\text {prop }}\right)$.

The acoustic measurements are performed from polar angle $\theta=30^{\circ}$ to $\theta=120^{\circ}$, with increments of $10^{\circ}$. The polar angle is measured from the jet downstream direction.

The acoustic measurements provided by Dr. Bridges from the NASA Glenn Research Center are in the format of lossless spectra per resolution bandwidth and "back" propagated to a distance of $0.3 \mathrm{~m}$. Thus, before performing the acoustic measurements comparison from two facilities, the received data from NASA were transformed to non-dimensionalized frequency and propagated back to $R_{\text {prop }}=100 \mathrm{D}$.

Finally, the OASPL is calculated for both sets of data using the same non-dimensionalized frequency range in order to obtain an unbiased comparison.

\section{Presentation of results}

The matrix of operating conditions of the data acquired at both PSU and NASA is shown in Fig. 4 and summarized in Table 2. In Fig.4, $M a_{\mathrm{a}}$ is acoustic Mach number. In Table 2, the jet Mach number $M a_{\mathrm{j}}$ relates to the average Mach number

Table 2 Operating conditions of unheated jets, PSU simulated heated jets and NASA heated jets.

\begin{tabular}{|c|c|c|c|c|c|c|}
\hline \multirow[b]{2}{*}{ NPR } & \multirow[b]{2}{*}{$M a_{\mathrm{j}}$} & \multicolumn{3}{|c|}{ PSU unheated jets } & \multicolumn{2}{|c|}{ NASA unheated jets } \\
\hline & & TTR & $M a_{\mathrm{a}}$ & $R e$ & $\mathrm{TTR}_{\text {mix }} / \mathrm{TTR}_{\text {core }}$ & $M a_{\mathrm{ami}}$ \\
\hline 2.5 & 1.22 & 1 & 1.07 & 595400 & 1 & 1.07 \\
\hline 3.0 & 1.36 & 1 & 1.16 & 727800 & 1 & 1.16 \\
\hline 3.5 & 1.47 & 1 & 1.23 & 857200 & 1 & 1.23 \\
\hline 4.0 & 1.56 & 1 & 1.28 & 989000 & 1 & 1.28 \\
\hline \multirow[t]{2}{*}{4.5} & 1.64 & 1 & 1.32 & 1119700 & 1 & 1.32 \\
\hline & & \multicolumn{3}{|c|}{ PSU simulated heated jets } & \multicolumn{2}{|l|}{ NASA heated jets } \\
\hline NPR & $M a_{\mathrm{j}}$ & TTR & $M a_{\mathrm{a}}$ & $R e$ & $\mathrm{TTR}_{\text {mix }} / \mathrm{TTR}_{\text {core }}$ & $M a_{\mathrm{ami}}$ \\
\hline \multirow[t]{2}{*}{3.0} & 1.36 & 2.56 & 1.86 & 499500 & $2.56 / 3.00$ & 1.86 \\
\hline & & 3.00 & 2.01 & 432300 & & \\
\hline \multirow[t]{2}{*}{3.5} & 1.47 & 3.00 & 2.14 & 553400 & $3.00 / 3.60$ & 2.13 \\
\hline & & 3.6 & 2.33 & 470800 & & \\
\hline \multirow[t]{2}{*}{4.0} & 1.56 & 3.00 & 2.22 & 636600 & $3.00 / 3.60$ & 2.22 \\
\hline & & 3.60 & 2.43 & 544500 & & \\
\hline \multirow[t]{2}{*}{4.5} & 1.64 & 3.00 & 2.29 & 723300 & $3.00 / 3.60$ & 2.29 \\
\hline & & 3.60 & 2.51 & 617000 & & \\
\hline
\end{tabular}




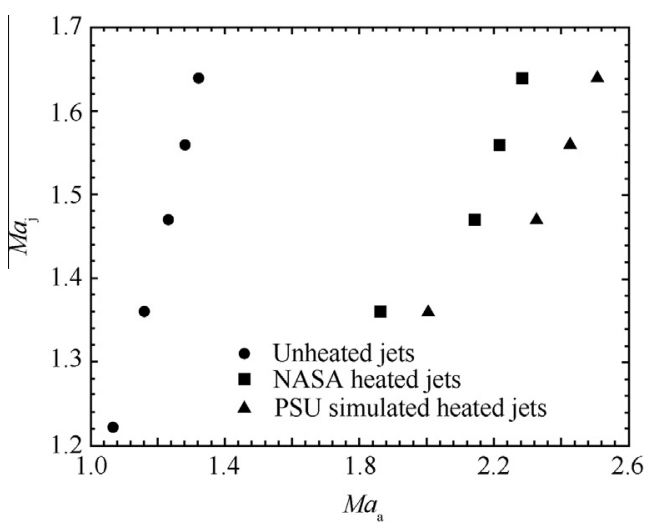

Fig. 4 Condition matrix of unheated and heated jets in acoustic Mach number vs jet Mach number.

of the fully expanded jet and is uniquely defined by the nozzle pressure ratio (NPR) and the nozzle total temperature ratio (TTR) defined from the jet stagnation temperature $T_{0}$ and the ambient temperature $T_{\infty}$ by TTR $=T_{0} / T_{\infty}$. For the data from NASA, the subscript "core" is used to refer to quantities relative to the core of the jet, while the subscript "mix" relates to the quantities relative to the mixture of the annular and core flow. The values of Reynolds number in Table 2 were computed based on the $M a_{\mathrm{d}}=1.3(\mathrm{AR}=1.067) \mathrm{GE}$ nozzle and following the calculation methodology described by Doty and McLaughlin. ${ }^{1}$ In addition to the three GE nozzles, a contoured CD nozzle designed to operate fully expanded at a design Mach number $M a_{\mathrm{d}}=1.5$ was used for qualification of the spectral results. A series of unheated and simulated heated jet experiments were conducted at PSU. As a first step, comparisons were made between cold measurements obtained at PSU with similar measurements from NASA. These are presented in the first section of the results. Then, the helium simulated small-scale results are compared in the second section of the results with the medium-scale hot jet measurements provided by NASA, with discussions of the results included.

\subsection{Unheated jets experiment}

Firstly, comparisons are made between acoustic spectra from cold jets, between small-scale measurements acquired at PSU and medium-scale measurements from NASA. This allows for a direct cross-scale comparison, from jets issuing from nozzles of different sizes but exactly the same geometry, without adding the complication of the heat simulation. Sample spectra are shown in Fig. 5 for two operating conditions: both jets are operated with a pressure ratio NPR $=4$, one of them issuing from the $M a_{\mathrm{d}}=1.3(\mathrm{AR}=1.067) \mathrm{GE}$ nozzle and the other from the $M a_{\mathrm{d}}=1.65(\mathrm{AR}=1.295) \mathrm{GE}$ nozzle. In Fig. 5, the black curves represent data acquired at PSU, with nozzles about $1.8 \mathrm{~cm}$ in diameter, and the light grey curves are spectra acquired at NASA with nozzles seven times larger. There is a very good agreement between the data acquired from the two facilities, providing a check on the scaling methodology and validating the quality of cold jet measurements acquired from both facilities (The level of screech tones is relatively higher in NASA's data than in PSU's data. The magnitude of screech tones is related to the different upstream flow boundary layer conditions from various jet facilities and the differences on the nozzle lip thickness. However, this is not critical since screech is not observed in the actual engine operation). This is an encouraging comparison which validates the potential to develop noise reduction concepts in small-scale jets that accurately simulate the acoustics issuing from the actual engine exhausts.

Closer inspection of the details of the small-scale nozzles used in conducting the experiments at PSU shows that the internal nozzle surface is slightly rougher at this scale level in

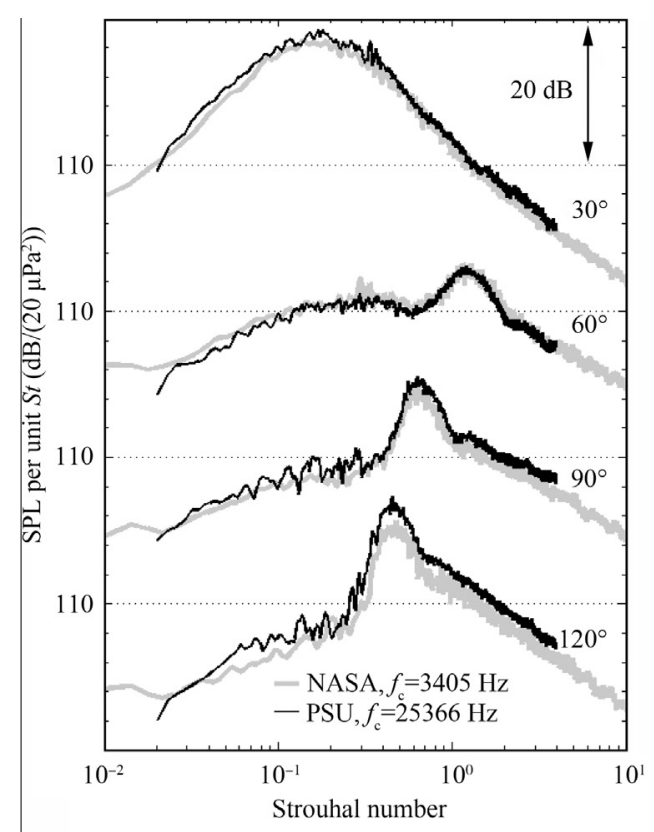

(a) Spectral from $M a_{\mathrm{d}}=1.3 \mathrm{GE}$ nozzle

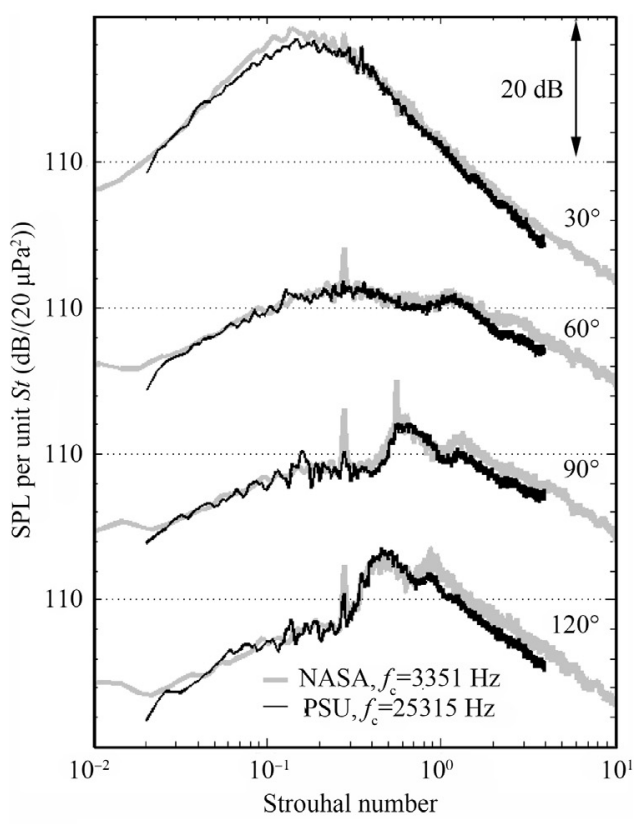

(b) Spectral from $M a_{\mathrm{d}}=1.65 \mathrm{GE}$ nozzle

Fig. 5 Spectral and OASPL comparison of unheated jets from PSU and NASA issuing from $M a_{\mathrm{d}}=1.3(\mathrm{AR}=1.067) \mathrm{GE}$ nozzle and $M a_{\mathrm{d}}=1.65(\mathrm{AR}=1.295) \mathrm{GE}$ nozzle both operated at $\mathrm{NPR}=4, M a_{\mathrm{j}}=1.56$. 


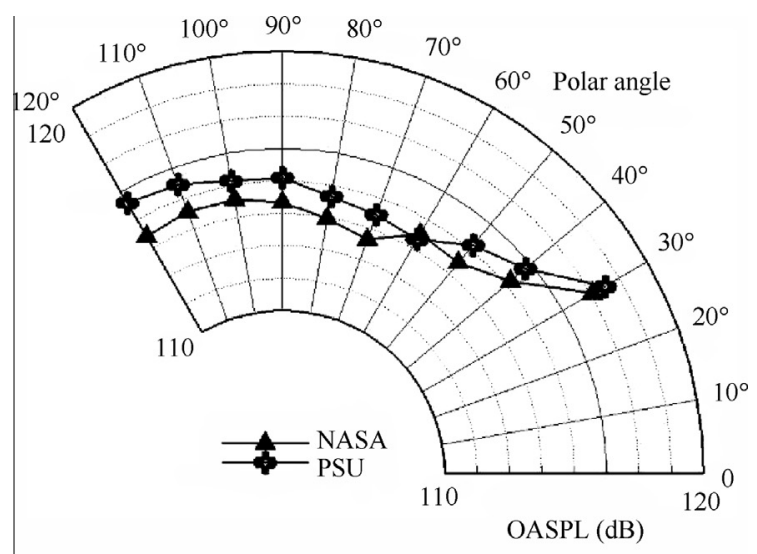

(c) OASPL from $M a_{\mathrm{d}}=1.3 \mathrm{GE}$ nozzle

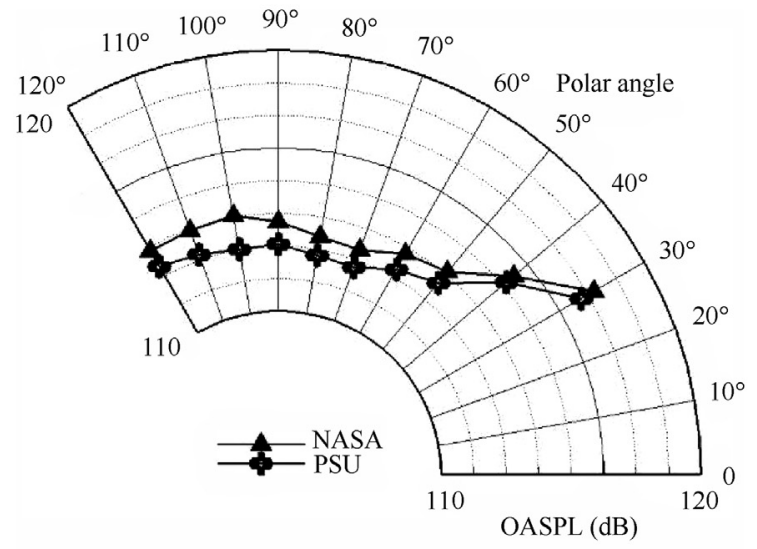

(d) OASPL from $M a_{\mathrm{d}}=1.65 \mathrm{GE}$ nozzle

Fig. 5 (continued) comparison with the moderate-scale nozzle. The lip thickness is also not exactly reproduced, due to the limitations of the rapid prototyping technique and the brittleness of the material used. Therefore, the thickness of the boundary layer at the exit plane of the nozzle may not scale perfectly with the moderatescale measurements. In spite of these differences, the comparison results are very good. This observation is in agreement with a previous study from Viswanathan and Clark ${ }^{8}$ who conducted experiments in order to validate the effect of the state of the flow at the nozzle exit plane. The conclusion from that study is that the radiated noise is insensitive to the state of the flow and the thickness of the boundary layer at the nozzle exit plane, provided that the Reynolds number of the jet flow exceeds approximately 400000.

In order to observe the effect on the radiated noise of the detailed shape of the GE nozzle, comparison to contoured CD nozzle experiments were conducted at PSU. Recall that the GE design has 12 facets around the perimeter and straight walls in the flow direction in both the converging and diverging sections. Spectral comparisons are shown in Figs. 6-8 between the contoured $M a_{\mathrm{d}}=1.5 \mathrm{CD}$ nozzle and the GE nozzle with a design Mach number $M a_{\mathrm{d}}=1.5(\mathrm{AR}=1.18)$. While acoustic measurements were gathered from $\theta=30^{\circ}$ to $\theta=120^{\circ}$, the main differences between the noises generated by the two nozzles appear in the broadband shock associated noise (BBSAN). This noise component is only (strongly) apparent at angles sideline to the jet and in the forward quadrant (i.e., $\theta>90^{\circ}$ ). Therefore, only spectra at $\theta=90^{\circ}$ and $\theta=120^{\circ}$ are shown here. Schlieren images of the jets are also presented next to the spectra in order to obtain some qualitative information of the flows. Comparisons are first shown for an over-expanded jet in Fig. 6. The schlieren images show a maximum of $10 \%$ discrepancy between the shock cell lengths, and the spectra from the two jets compare fairly well with no significant

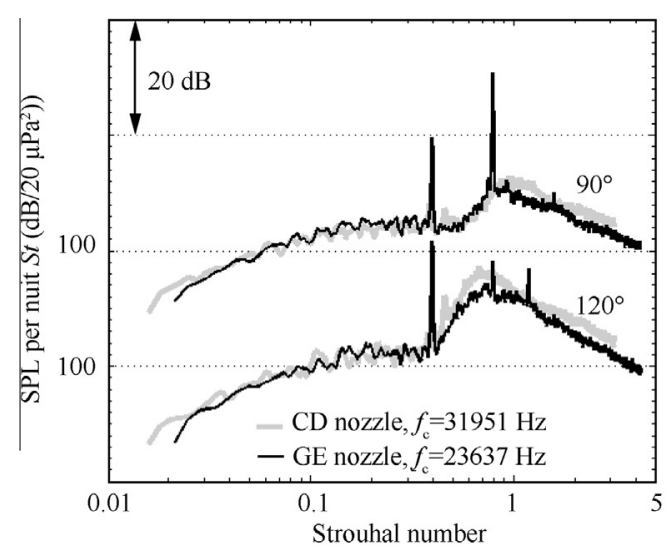

(a) Spectra of unheated jets

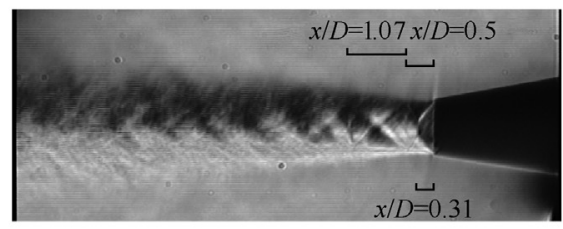

(b) Schelieren image of CD nozzle

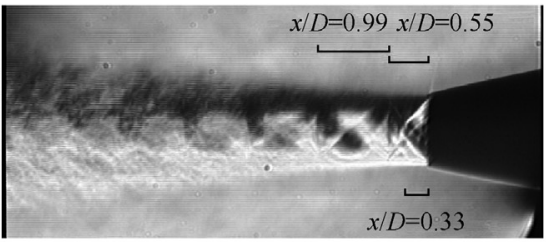

(c) Schelieren of image of GE nozzle

Fig. 6 Spectra of unheated jets from CD and GE nozzles (both with $M a_{\mathrm{d}}=1.5, \mathrm{AR}=1.18$ ) operated at $M a_{\mathrm{j}}=1.3$ conducted at PSU, schlieren images of CD nozzle and GE nozzle. 


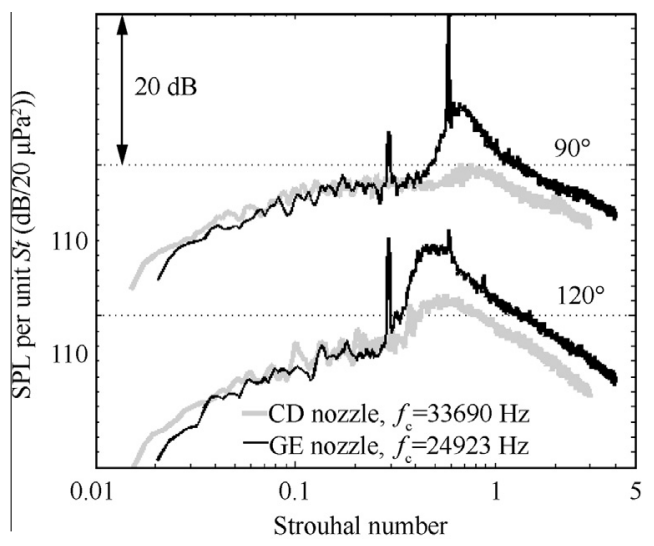

(a) Spectra of unheated jets

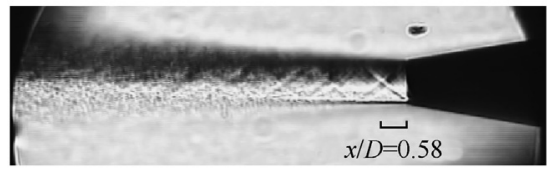

(b) Schelieren image of CD nozzle

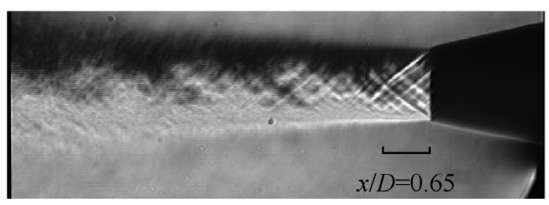

(c) Schelieren of image of GE nozzle

Fig. 7 Spectra of unheated jets from CD and GE nozzles (both with $\left.M a_{\mathrm{d}}=1.5, \mathrm{AR}=1.18\right)$ operated at $M a_{\mathrm{j}}=1.5$ conducted at PSU, schlieren images of CD nozzle and GE nozzle.

discrepancy. This is consistent with the general belief that alternative nozzle shapes do not significantly alter the generated sound in over-expanded conditions. In such conditions, the jet exhausting from both nozzles needs to go through an oblique shock (possibly a strong normal shock) in order to balance the exit pressure to the ambient pressure. Thus, it is reasonable that the spectra are close to each other at the polar angles of $90^{\circ}$ and $120^{\circ}$ since the jet exhausts consist of similar flow fields. Fig. 7 shows the comparison obtained from both nozzles operating a fully expanded $M a_{\mathrm{j}}=1.5 \mathrm{jet}$. The schlieren visualizations reveal the presence of shocks in both nozzles, but they are not as strong as in the case of the CD nozzle jet (designed to operate shock-free at this specific pressure ratio). A large number of weak shock waves can be seen in the jet issuing from the GE nozzle, due to the presence of facets and the conical design of the diverging section. Since there are more shock waves in this flow, it is not surprising to observe that the BBSAN part of the spectra is louder by about $8 \mathrm{~dB}$. Fig. 8 shows both nozzles operating at an under-expanded condition. In this comparison, the shock cell lengths are very similar between the two flows. As a result, the spectra from both jets match very well. Furthermore, the screech tone present in the CD nozzle is eliminated with the GE design. In typical aircraft operating conditions, the variable-geometry nozzles are usually operating at under-expanded conditions for flow stability considerations. Since the effect on the noise spectra is negligible under such conditions when compared to a CD nozzle, the facetted conical design appears to be an appropriate aeroacoustic design.

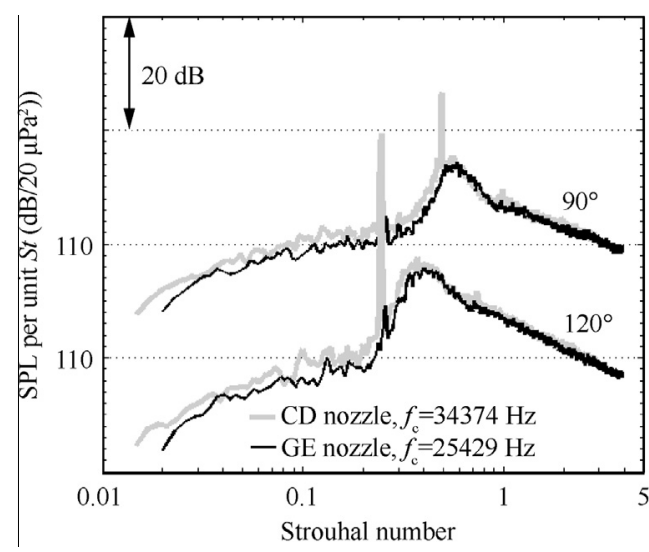

(a) Spectra of unheated jets

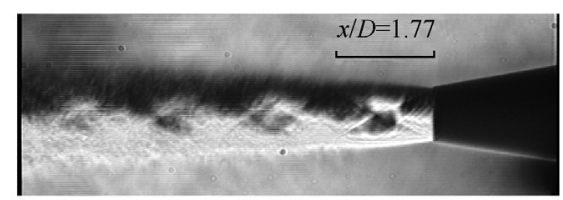

(b) Schelieren of image of $\mathrm{CD}$ nozzle

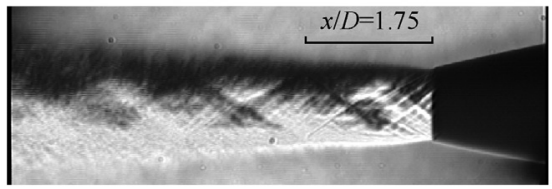

(c) Schelieren of image of GE nozzle

Fig. 8 Spectra of unheated jets from CD and GE nozzles (both with $\left.M a_{\mathrm{d}}=1.5, \mathrm{AR}=1.18\right)$ operated at $M a_{\mathrm{j}}=1.7$ conducted at PSU, schlieren images of CD nozzle and GE nozzle.

Finally, an overview of Figs. 6-8 shows the typical trend of screech frequency decreasing with increasing Mach number, even though the screech tones do not appear for all conditions with both nozzles. These screech frequencies are consistent with analytical predictions, as described by Tam et al. ${ }^{9}$ Again, the difference on the appearance of screech tones is not critical because screech is not observed in the actual engine operation.

\subsection{Heated jets experiment}

In an attempt to further qualify the data from both facilities and produce data relevant to a full-scale engine, heated jet operating conditions are investigated. The simulated heated jets experiments were conducted via helium-air mixture jets at PSU, following the methodology established during previous studies ${ }^{1,6}$ and briefly described in the prior section of this paper.

Figs. 9-12 show acoustic spectra measured with the GE design nozzles with all three AR values and conducted at both PSU and NASA. For the NASA experiments, a small amount of cold bleed flow surrounds the core flow (of temperature ratio $\mathrm{TTR}_{\text {core }}$ ) and after some mixing yields a mixed flow temperature ratio $\mathrm{TTR}_{\text {mix }}$. The mixed flow temperature ratio $\mathrm{TTR}_{\text {mix }}$ is the simulated temperature ratio chosen for the PSU heliumair mixture jets. For the data shown in Fig. 9, the data recorded using the $M a_{\mathrm{d}}=1.3(\mathrm{AR}=1.067) \mathrm{GE}$ nozzle operating at a pressure ratio of NPR $=3.5$ produces an average exit Mach number $M a_{\mathrm{j}}=1.47$, and the simulated temperature 


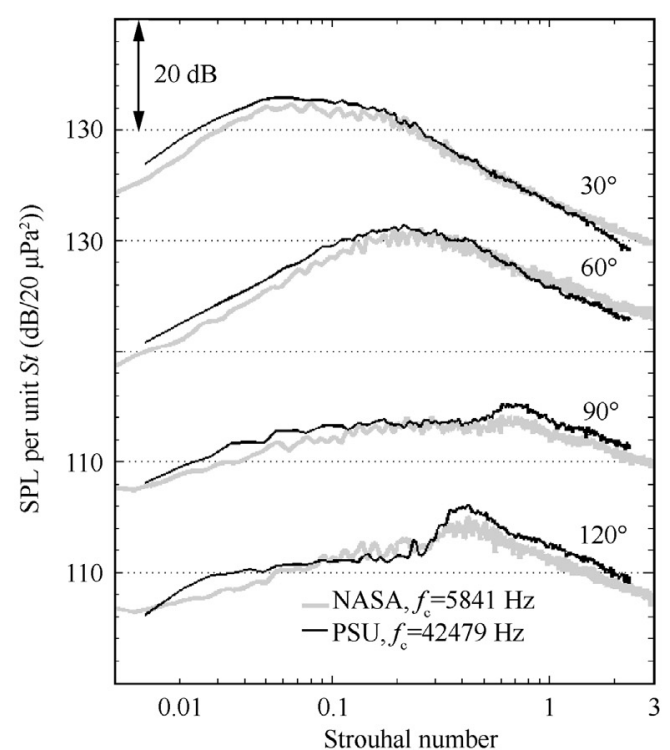

(a) Spectra from $M a_{\mathrm{d}}=1.3 \mathrm{GE}$ nozzle

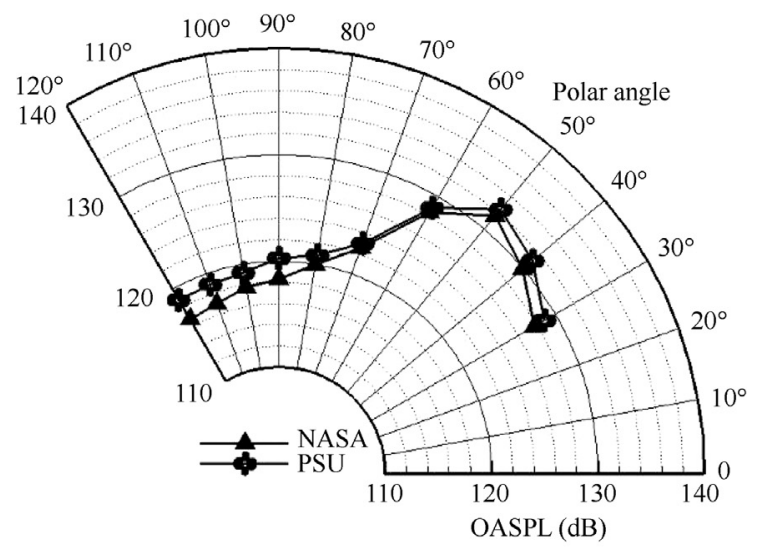

(b) OASPL from $M a_{\mathrm{d}}=1.3 \mathrm{GE}$ nozzle

Fig. 9 Spectral and OASPL comparison of simulated heated jets $(\mathrm{TTR}=3.0)$ from PSU and heated jets $\left(\mathrm{TTR}_{\operatorname{mix}}=3.0\right)$ from NASA both issuing from $M a_{\mathrm{d}}=1.3(\mathrm{AR}=1.067) \mathrm{GE}$ nozzle operated at NPR $=3.5, M a_{\mathrm{j}}=1.47$.

ratio $\mathrm{TTR}=3.0$ for PSU and $\mathrm{TTR}_{\text {mix }}=3.0\left(\mathrm{TTR}_{\text {core }}=3.6\right)$ for NASA. For the data shown in Fig. 10, the $M a_{\mathrm{d}}=1.5$ $(\mathrm{AR}=1.18) \mathrm{GE}$ nozzle was used at both PSU and NASA with a pressure ratio of NPR $=4.5$. This yields a jet Mach number of 1.64 issuing from a nozzle whose AR corresponds to an $M a_{\mathrm{j}}=1.5$ jet. In this case, the PSU jet was operated at a total temperature ratio of TTR $=3.0$ corresponding to the NASA mixed flow temperature of the same value. The NASA core temperature ratio was 3.6 for this condition.

The data for the small-scale experiments compare very favorably with the NASA moderate-scale data, across the polar angle range (in the case of the OASPL plot) and across the frequency range at the specific polar angles shown. To reiterate, the experiments have been conducted with non-dimensionally identical nozzle contours and identical pressure ratios (both to engineering accuracy). The mixture simulated total temperature ratio of the PSU small-scale measurements matches the mixture total temperature ratio of the NASA hot jet. There is some uncertainty however in using this mixed

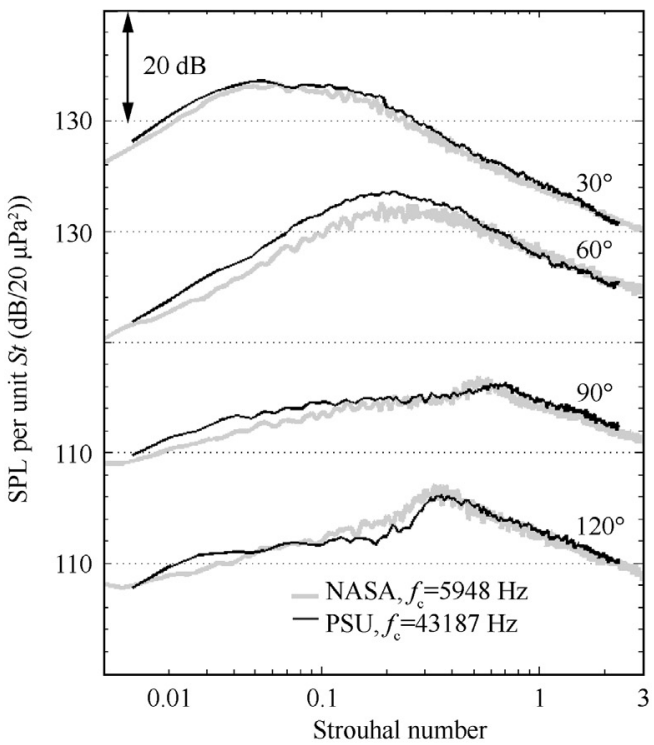

(a) Spectra from $M a_{\mathrm{d}}=1.5 \mathrm{GE}$ nozzle

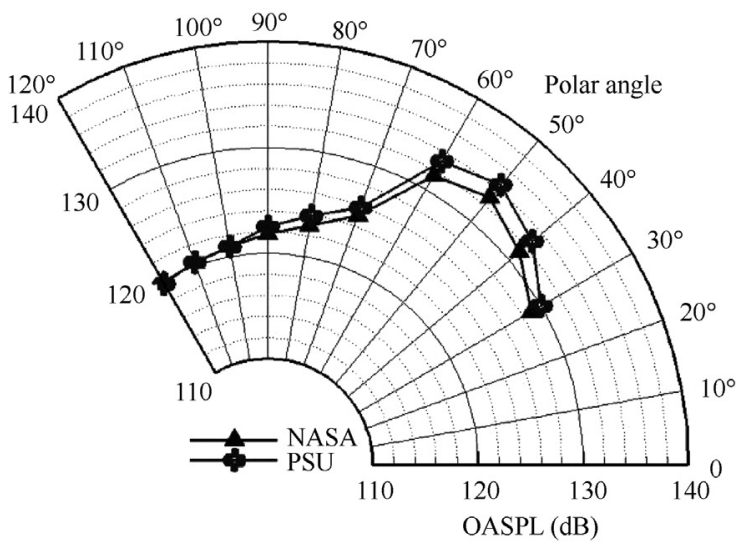

(b) OASPL from $M a_{\mathrm{d}}=1.5 \mathrm{GE}$ nozzle

Fig. 10 Spectral and OASPL comparison of simulated heated jets $($ TTR $=3.0)$ from PSU and heated jets $\left(\right.$ TTR $\left._{\text {mix }}=3.0\right)$ from NASA both issuing from $M a_{\mathrm{d}}=1.5(\mathrm{AR}=1.18) \mathrm{GE}$ nozzle operated at NPR $=4.5, M a_{\mathrm{j}}=1.64$.

flow total temperature ratio in favor of the core total temperature ratio based on the assumption that the cold jet annulus simply cools the jet nozzle walls. If this core temperature ratio is used as the basis of comparison for setting the PSU simulated temperature ratio, the small-scale acoustic data are higher than the NASA data by as much as $5 \mathrm{~dB}$ in the low frequency regime $(S t<0.2)$ of the spectrum.

Two additional comparisons were conducted in an effort to evaluate the importance of matching the acoustic Mach number $M a_{\mathrm{a}}$ obtained by using the mixed flow total temperature ratio to represent the NASA jet temperature. Fig. 11 shows the comparison of the acoustic measurements made using the $M a_{\mathrm{d}}=1.5(\mathrm{AR}=1.18) \mathrm{GE}$ nozzle operated at $M a_{\mathrm{a}}=2.3$ where the operating condition is $M a_{\mathrm{j}}=1.467$ and $\mathrm{TTR}=3.6$ for the PSU experiments, and the operating condition is $M a_{\mathrm{j}}=1.638$ and $\mathrm{TTR}_{\text {mix }}=3.0$ for NASA. Jets with the same acoustic Mach number $M a_{\mathrm{a}}$, should produce similar sound intensity, particularly in the downstream arc where Mach wave radiation dominates the noise. On the sideline 


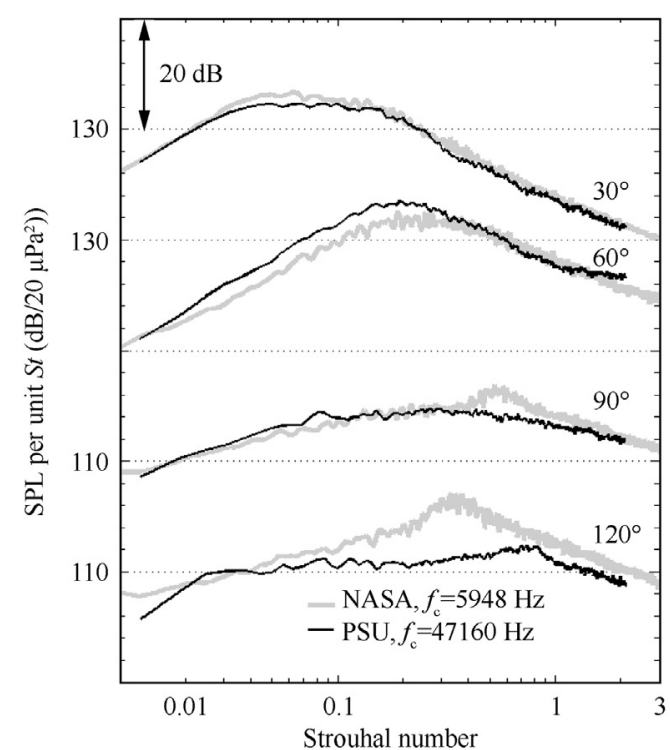

(a) Spectra from $M a_{\mathrm{d}}=1.65 \mathrm{GE}$ nozzle

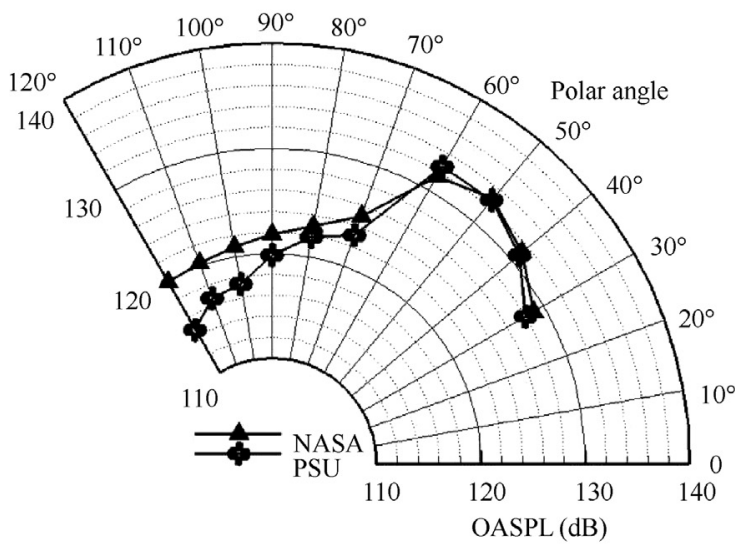

(b) OASPL from $M a_{d}=1.5$ GE nozzle

Fig. 11 Spectral and OASPL comparison in matching acoustic velocity from simulated heated jets (TTR $=3.6$ ) of PSU operated at $\mathrm{NPR}=3.5, \quad M a_{\mathrm{j}}=1.467, \quad M a_{\mathrm{a}}=2.3$ and heated jets $\left(\mathrm{TTR}_{\text {mix }}=3.0\right)$ of NASA operated at NPR $=4.5, M a_{\mathrm{j}}=1.638$, $M a_{\text {amix }}=2.3$ both with $M a_{\mathrm{d}}=1.5(\mathrm{AR}=1.18) \mathrm{GE}$ nozzle.

the NASA data have significant broadband shock noise as expected because of the significantly off-design pressure ratio.

The data shown in Fig. 12 show a similar comparison except this time the nozzle chosen has an AR of 1.295, with a corresponding design Mach number of $M a_{\mathrm{d}}=1.65$. In this case the NASA model jet is nearly perfectly expanded and no shock noise is apparent. The corresponding PSU jet is running overexpanded and the resulting shock noise is unexplainably absent until $\theta=90^{\circ}$

At this point, one can see that there are conditions in which the acoustic data measured during the small-scale experiments replicate the NASA moderate-scale data very closely. The cold jet data are all within experimental uncertainty at virtually every polar angle and frequency, except for the extent of shock screech which is more apparent in the small-scale experiments. In the heat simulated jets, the shock screech is not a problem but there remains uncertainty in how the thin annulus of room temperature air surrounding the hot core jet should be

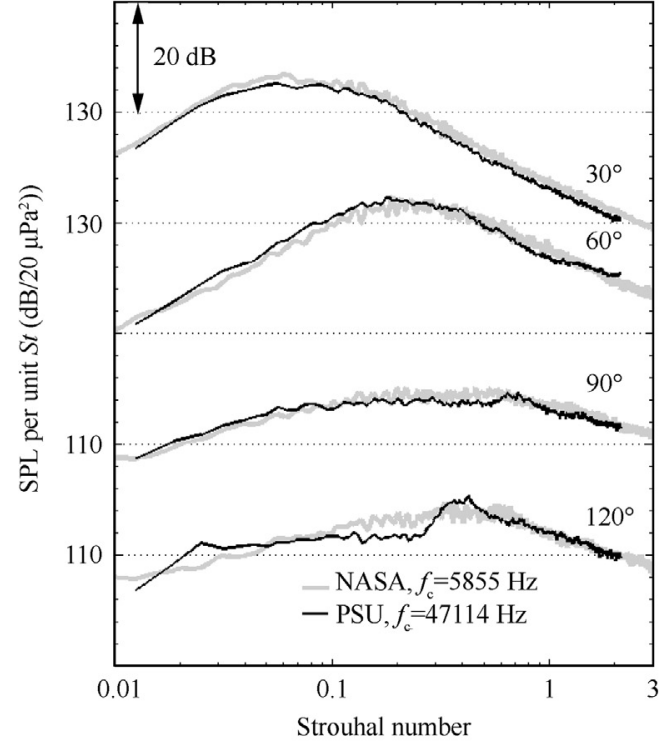

(a) Spectra from $M a_{\mathrm{d}}=1.65 \mathrm{GE}$ nozzle

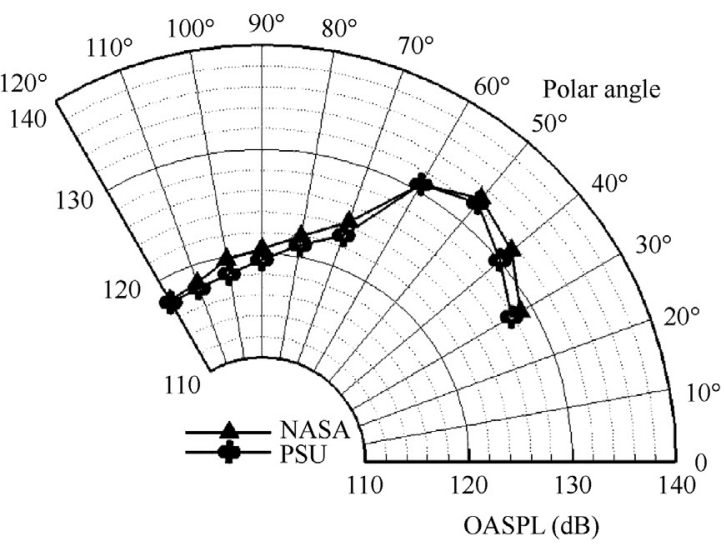

(b) OASPL from $M a_{\mathrm{d}}=1.65 \mathrm{GE}$ nozzle

Fig. 12 Spectral and OASPL comparison in matching acoustic velocity from simulated heated jets (TTR $=3.6$ ) of PSU operated at $\mathrm{NPR}=3.5, \quad M a_{\mathrm{j}}=1.467, \quad M a_{a}=2.3$ and heated jets $\left(\mathrm{TTR}_{\text {mix }}=3.0\right)$ of NASA operated at NPR $=4.5, M a_{\mathrm{j}}=1.638$, $M a_{\text {amix }}=2.3$ both with $M a_{\mathrm{d}}=1.65(\mathrm{AR}=1.295)$ GE nozzle.

modeled by the smaller-scale helium-air mixture jet experiments. This issue is the subject of ongoing experiments.

\section{Conclusions}

The acoustic measurements of models of military style supersonic nozzles were conducted in unheated and simulated heated jets. The characteristic of multi-facetted inside conical contour from the military style nozzle generates disparate flow fields operating in unheated jets in comparison with the contoured CD nozzle. The major difference between two nozzles is the excess broadband shock associated noise issuing from the military style nozzle, and the consequence is the same as the theoretical conjecture. In typical aircraft operating conditions, the variable-geometry nozzles usually operate at under-expanded conditions for flow stability considerations. Since the effect on the noise spectra is negligible under such 
conditions when compared to a CD nozzle, the facetted conical design appears to be an appropriate aeroacoustic design.

The cross-scale comparison methodology was deployed to produce direct comparisons between the small-scale measurements made at PSU and the medium-scale measurements from the NASA Glenn Research Center. The comparison of various scales unheated jets operating at the same condition shows that they identically match to each other. The details of the smallscale nozzles used in conducting the experiments at PSU show that the internal nozzle surface is slightly rougher at this scale level in comparison with the moderate-scale nozzle. In addition, the lip thickness is not exactly reproduced, due to the limitations of the rapid prototyping technique and the brittleness of the material used. Therefore, the thickness of the boundary layer at the exit plane of the nozzle may not scale perfectly with the moderate-scale measurements. In spite of these differences, the comparison results are very good. Then, the comparison of various scales heated jets operating at the same condition shows good agreement within maximum $2 \mathrm{~dB}$ deviation. Meanwhile, the comparison of various scales jets operating at the same acoustic Mach number also shows that they match pretty well. The current discrepancies can be attributed either to the heat simulation or to the presence of the cold annular flow for the NASA data. More experiments and comparisons will be needed to discriminate between these two. Averagely, the cross-scale comparison methodology exhibits that the small-scale simulated heated jets can adequately simulate the acoustics issuing from the moderate-scale heated jets. The novel concept of sound suppression mechanism can be deployed in the small-scale jets to well predict the acoustics issuing from the actual engine exhausts.

\section{Acknowledgements}

This work was performed under the sponsorship of the Strategic Environmental Research and Development Program, project number WP-1583. The authors acknowledge Dr. Bridges for the supporting data from the NASA Glenn Research Center, and Prof. Morris of Pennsylvania State University for his consulting with this work.

\section{References}

1. Doty MJ, McLaughlin DK. Acoustic and mean flow measurements of high speed helium air mixture jets. Int $J$ Aeroacoustics 2003;2(2):293-334.

2. Veltin $\mathbf{J}$, McLaughlin DK. Noise mechanisms investigation in shock containing screeching jets using optical deflectometry. In: Proceedings of 14th AIAA/CEAS aeroacoustics conference; 2008.

3. Kuo C-W, Veltin J, McLaughlin DK. Methods to improve the accuracy of acoustic measurements in small scale high speed jets. In: Proceedings of 15th AIAA/CEAS aeroacoustics conference; 2009.

4. Kuo C-W, Veltin J, McLaughlin DK. Effects of jet noise source distribution on acoustic far-field measurements. Int $J$ Aeroacoustics 2012;11(7):885-915.

5. Bridges J, Brown CA. Validation of the small hot jet acoustic rig for aeroacoustic research. In: Proceedings of 11th AIAA/CEAS aeroacoustics conference; 2005.

6. Papamoschou D. Acoustic simulation of coaxial hot air jets using cold helium-air mixture jets. J Prop Power 2007;23(2):375-81.

7. Kinzie KW, McLaughlin DK. Measurements of supersonic helium/ air mixture jets. AIAA J 1999;37(11):1363-9.

8. Viswanathan K, Clark LT. Effect of nozzle internal contour on jet aeroacoustics. Int J Aeroacoustics 2004:3(2):103-35.

9. Tam CKW, Seiner JM, Yu JC. Proposed relationship between broadband shock associated noise and screech tones. J Sound Vibr 1986;110(2):309-21.

Ching-Wen Kuo is a research associate in the Department of Aerospace Engineering at Pennsylvania State University. He is mainly focused on experimental investigation and analysis for noise generation, radiation and suppression mechanisms in supersonic jets. 